\title{
Miniaturized Microstrip Diplexer Based on FR4 Substrate for Wireless Communications
}

\author{
Hussein Ali Hussein ${ }^{1}$, Yaqeen Sabah Mezaal ${ }^{2,}$, Ban M. Alameri ${ }^{3}$ \\ ${ }^{1}$ Department of Electrical Engineering, University of Technology, \\ Baghdad, Iraq \\ ${ }^{2}$ Medical Instrumentation Engineering Department, Al-Esraa University College, \\ Baghdad, Iraq \\ ${ }^{3}$ Department of Electrical Engineering, Faculty of Engineering, Mustansiriyah University, \\ Baghdad, Iraq \\ yaqeen@esraa.edu.iq
}

\begin{abstract}
In this paper, the miniaturized microstrip diplexer has been designed for dual channels based on FR4 substrate material. It consists of two bandpass filters (BPFs) functioning under dissimilar frequency bands coupled with a unified junction. Every BPF has been created by a meandered line resonator, step impedance resonator, uniform impedance resonator, and input/output feed lines. The AWR electromagnetic simulator has been used for characterizing the frequency responses of the projected diplexer. Noble scattering parameter results with narrow band responses and negative group delay values are obtained for the proposed diplexer. The microstrip diplexer has an interesting band isolation between the two filters around $31 \mathrm{~dB}$. The device has been successfully fabricated and verified with the simulations.
\end{abstract}

Index Terms-Diplexer; Microstrip; FR4 material; Bandpass filter; SIR; UIR; Narrow band response; Negative group delay; Electronic design automation (EDA).

\section{INTRODUCTION}

Multiplexers and diplexers play a role in many different communication systems. For example, radio transmission, satellite-communication, and broadband wireless communications. The simplest form of the multiplexer is the diplexer. Two transmitters on diverse channels can use a popular antenna at the same time by utilizing the diplexer. Diplexer technologies are used in both base station and radio handsets in cellular radio systems for many system standards. Figure 1 depicts a layout of the front end of a cellular base station. The diplexer consists of the power divider and two filters with severe specifications on selectivity and isolation. Ordinarily, the transmitter creates signals with comparatively high power, and the transmit filter must be able to handle such a high power. In contrast, the receiver should be capable to detect extremely weak signals [1].

A lot of interest has been observed in designing microwave diplexer, because of its capability for combining two different filters in one device using communication channels. The complexity reduction and miniaturization for microwave and millimetre wave devices with high quality

Manuscript received 21 March, 2021; accepted 16 June, 2021. performance are requested by the scholars in this area of research. These specifications make the diplexer efficient in terms of cost and size with separate channels. The diplexer can be defined as a device to be used either for combing dual sub-band into single common wide band, or dividing the frequency band into dual separated sub-bands. Generally speaking, the microwave diplexers are employed to connect two networks with diverse in service frequencies to the common port [2].

In this research article, a new compact microstrip diplexer based on meander line resonators, uniform impedance resonator, and step impedance resonators has been designed using FR4 epoxy laminate material. As compared to many reported papers in the literature, the projected diplexer has considerable compactness, narrow band frequency responses, and efficient band isolations around $31 \mathrm{~dB}$ among the frequency channels under tolerable insertion loss and return loss parameters.

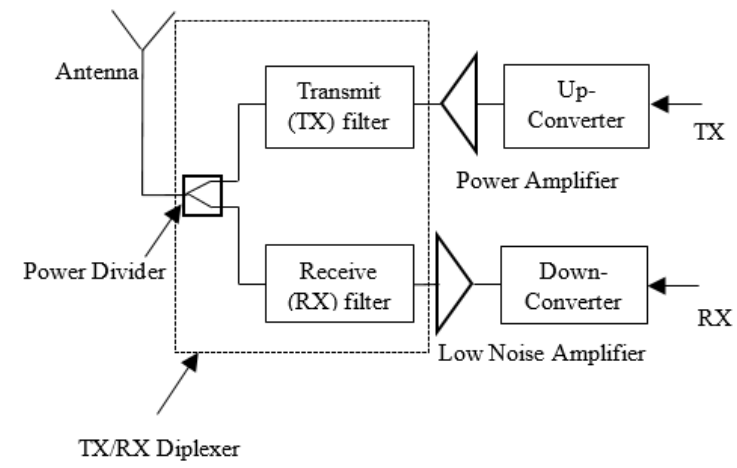

Fig. 1. RF front end for cellular base station.

\section{RELATED WORKS}

In [3], the study discusses different diplexer designs for the corrugated and quasi-optical waveguide transmission systems. It is based on high power tests, low power experiments, and numerical calculations at Electron Cyclotron Resonance Heating (ECRH) system to show the ability of these devices. In [4], two layouts to achieve miniaturized microwave diplexers in two mode cavities 
have been proposed. One of them is a traditional one. The joint port has been connected to the dual resonators in the detached paths. The other layout is original due to a connection of one resonator by a mutual port. The spurious cross-polarized modes are removed on diplexers of dual mode cavity by the second topology, whereas these modes are not eliminated by the first one. The authors in [5] presented a multiband filter employing the mushroom-like crack-upper structure on the dual layers of the electromagnetic band gap and transmission line. The projected filter is used in the diplexer design. The CST software was adopted to demonstrate the parameters of this diplexer which has given an interesting electrical performance.

The authors in [6] have presented the design steps and implementing of a diplexer in the printed circuit layout. The diplexer had been designed by two filters that have various bandpass behaviours, where the input ports had been jointed as diplexer input and the output ports of the two filters as diplexer out port. The authors in [7] proposed the E-Band diplexers to be employed as a front-end equipment in the point to point wireless communication systems. They have been dedicated to operate at a frequency spectrum of $81 \mathrm{GHz}-86 \mathrm{GHz}$ and $71 \mathrm{GHz}-76 \mathrm{GHz}$. There are three topologies that are proposed. The first and the second ones are based on the traditional T-junction. The third one has a unique topology shaped from the coupled resonators. Two microstrip diplexers with multiband feature are introduced in [8]. The diplexers have the bandpass filters (BPFs) within the specified channels and feed lines. Every respective channel parameter is managed by one BPF, which is a result in high design flexibility. The microstrip with long feeding lines and high isolating characteristic support the channel filters. In [9], the researchers realized the microwave diplexer by swapping dual band BPFs into two single bands BPFs. This construction has eliminated the requirement for using exterior junctions in the diplexer topology, while the conventional diplexer has isolated junctions for energy allocation. A novel design for realizing microwave diplexer using groove gap waveguide resonators has been introduced in [10]. A high efficiency and economical Ku-band diplexer consisting of Chebyshev filters of $4^{\text {th }}$ order in TX and RX ends has been implemented. The simulation and measurement have verified the projected diplexer. In [11], a novel microstrip diplexer employing $\mathrm{H}$-shaped resonators with small dimensions and elevated band isolation has been introduced. This design has been operated under $2.4 \mathrm{GHz}$ for the Industrial Scientific Medical (ISM) application, and under $1.575 \mathrm{GHz}$, for the GPS application. The produced diplexer is achieved by joining dual bandpass filters by utilizing tapped microstrip Input/Output lines and H-shaped resonators. It presents an analytic theory of an equivalent LC circuit of the introduced bandpass filter with $\mathrm{H}$-model and the H-configured resonator. The dual cross-slots are dug in a base plane of a presented microstrip diplexer for enhancing its electrical behaviors and to achieve the pass bands at low frequencies. The circuit with the defected ground structure has been capable for L1 and L2 bands of the GPS spectrum. The frequency responses of the diplexer have been inspected by ADS simulator and proved with the
CST microwave Studio. A microwave diplexer design based on the substrate integrated waveguide (SIW) transmission line technology of the twenty-first century is proposed in [12]. A common junction was utilized to produce the diplexer employing an exterior junction for the energy allocation in the diplexer that usually raises the complication of the design and results in a huge equipment. The ability to develop diplexers by combining two and three resonator microstrip models, which are created with the elevated relative dielectric constant $\varepsilon_{\mathrm{r}}=80$ without short-circuited elements, is achieved in [13]. In [14], the authors proposed a diplexer with dual-channel utilizing microstrip open loop coupled resonators, where each of them has dual working band frequencies. The microstrip diplexer has considered $1.424 / 1.732 \mathrm{GHz}$ for the $1^{\text {st }}$ channel and $2.014 / 2.318 \mathrm{GHz}-$ for the $2^{\text {nd }}$ channel. An insertion loss of $1.8 \mathrm{~dB}$ and $1 \mathrm{~dB}$, and an insertion loss of $1.5 \mathrm{~dB}$ and $3 \mathrm{~dB}$ is achieved at load 1 and load 2, respectively. Moreover, it produces the return loss values higher than $10 \mathrm{~dB}$ with $35 \mathrm{~dB}$ of efficient isolation between the channels. The introduced layout has a simple topology, compact circuit dimensions, and narrowband frequency performances that are suitable for the wireless applications.

\section{DESIGN CONCEPT}

The Step Impedance Resonator (SIR) stands for resonant component in the Transverse Electromagnetic (TEM) or quasi TEM mode with two or more transmission lines and different characteristic impedances. SIR may be not valid for the microwave frequencies greater than $20 \mathrm{GHz}$ due to electromagnetic radiation, transverse resonance, and other negative characteristics. Nonetheless, the modern electromagnetic simulators and up-to-date microwave engineering technology should overcome this drawback. Due to their cost-effectiveness and simple topology, SIRs have been recognized in the RF and microwave models. They have less dimensions than the uniform impedance resonators (UIR), which are substantially adopted in RF and microwave devices [15]. The UIR and SIR instances are depicted in Fig. 2.

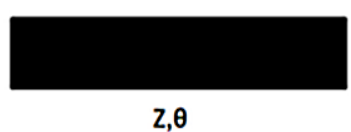

(a)

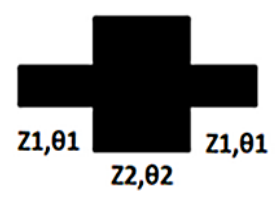

(b)

Fig. 2. (a) Uniform impedance resonator; (b) The equivalent step impedance resonators' configurations.

For UIR and its equivalent SIR configuration, the ABCD matrices will be the same with a lower physical SIR length than the UIR line [15]. UIR's ABCD matrix can be expressed as [15] 


$$
\left[\begin{array}{ll}
A & B \\
C & D
\end{array}\right]=\left[\begin{array}{cc}
\cos \theta & j Z_{o} \sin \theta \\
j Z_{o}^{-1} \sin \theta & \cos \theta
\end{array}\right]
$$

where $Z_{0}$ symbolizes the characteristic impedance, and $\boldsymbol{\theta}$ symbolizes the UIR line length. By interchanging it with three matrices in each segment of SIR, this matrix is feasibly used with SIR topology. As opposed to UIR topologies, many design parameters have been identified with SIR. Their resonance conditions in UIR topologies are based on their line lengths. On the other hand, when recognizing the resonance state of SIR, the length ratio and impedance ratio must be considered. In SIR, the resonance condition is specified by

$$
K=\tan \theta 1 \tan \theta 2 .
$$

Accordingly, "K" characterizes an impedance ratio coefficient that is equal to $Z 2 / Z 1$. By the way, $\theta 1$ and $\theta 2$ are the SIR electrical lengths. It was verified that for $0<K<1$, the electrical length for the resonator is the lowest and highest for $K>1$. However, for big magnitudes of $\mathrm{K}$, a huge discontinuity was observed in SIR that results in insufficient insertion loss [14].

The meandered line resonators stand for the planner layouts that can be fabricated straightforwardly. Essentially, these resonators have been extremely recognized for their superb behaviors and consolidated size; therefore, they are vastly employed as slowness lines and deviation systems. For that reason, they have been employed in the design of phase shifters, couplers, filters, and antennas. Figure 3(a) depicts the meander line resonator which can be shaped based on the sequences of LC lumped element circuit as presented in Fig. 3(b) [16].

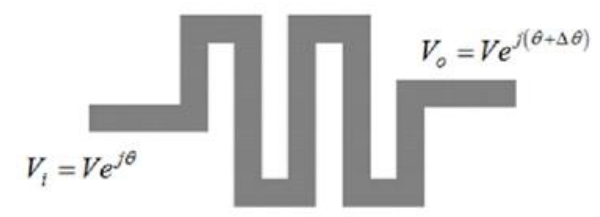

(a)

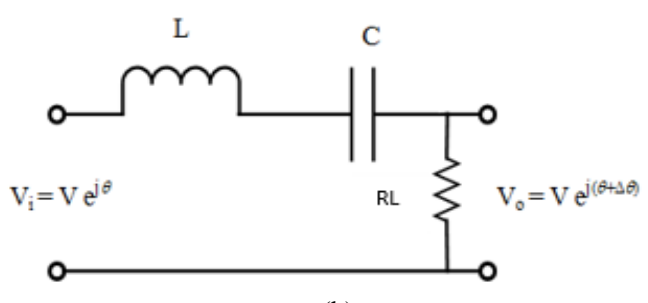

(b)

Fig. 3. (a) The classical layout of meandered line resonator; (b) The meandered line resonator lumped element circuit.

The impedance of the LC series according to Fig. 3(b) can be depicted on the form of $j(\omega L-1 / \omega C)$. Thus, at the end of the meander line resonator, the load is a resistance of RL, the phase delay can be expressed by [16]

$$
\Delta \theta=\tan ^{-1} \frac{\omega L-\frac{1}{\omega C}}{\mathrm{RL}} .
$$

The meandered line resonator has a phase constant which is

$$
\beta=\frac{2 \pi}{\lambda_{i}}
$$

where $\lambda_{i}$ stands for the resonator wavelength. According to the preceding equations, the following equation is written as

$$
\Delta \theta=\beta l
$$

or

$$
l=\Delta \theta / \beta=\frac{\lambda i}{2 \pi} \tan ^{-1} \frac{\omega L-\frac{1}{\omega C}}{\mathrm{RL}} .
$$

Therefore, (5) can be considered to plan this resonator. A series connection of $\mathrm{C}$ and $\mathrm{L}$ can be used to design the microstrip diplexer. With intended overall substrate size of diplexer $32 \times 26 \mathrm{~mm}^{2}$ using FR4 substrate with dielectric constant of 4.3 , thickness of $1.5 \mathrm{~mm}$, and loss tangent of 0.02 , the number of meander pins and dimensions of SIRs and UIRs were determined by the Applied Wave Research (AWR) simulator for designing, simulating, and optimizing the presented microstrip diplexer. Based on the Trial and Error concept and on the optimizer tools of the AWR simulator, the compact microstrip diplexer was intended to be operated under frequency channels of $1.855 \mathrm{GHz}$ and $2.505 \mathrm{GHz}$, respectively, with a high feasible band isolation and tolerable frequency responses. Figure 4 and Figure 5 show the details about the design elements and the assembling process. In addition to that, the space and the margin among the meander line resonators and the feed lines can adjust the simulator outcomes as well.

Namely, based on the meander resonators, SIR, and UIR elements as depicted in Fig. 4, the microstrip diplexer using FR4 substrate has been designed (see Fig. 5). The coupled junction was employed to connect the two filters, which have good features, such as the simplicity and the size reduction with an interested electrical performances. The gained size reduction is done by combining two filters in a single device instead of using two filters, more ports, or other related elements. The SIR elements are concentrated nearby SMA connectors and upper ends of I/O feeders. Actually joining the two filters creates the impairment of the required frequency response. As a result of that, an accurate optimization of each section of the circuit is required. The resonant frequency of the filter is determined by the length and the number of turns and UIR and SIR elements.

\section{Simulation Results AND Discussion}

The corresponding scattering parameter consequences for a diplexer based on FR4 substrate are illustrated in Fig. 6. The Tx channel has an insertion loss of $4.2 \mathrm{~dB}$, return loss of $32 \mathrm{~dB}$, and the ranged bandwidth from $1.82 \mathrm{GHz}$ to $1.881 \mathrm{GHz}$ at the center frequency of $1.855 \mathrm{GHz}$. At the center frequency of $2.505 \mathrm{GHz}$ in the $\mathrm{Rx}$ channel, the second channel is achieved with an insertion loss of $3.3 \mathrm{~dB}$ 
and return loss of $31 \mathrm{~dB}$ with the bandwidth range of $2.451 \mathrm{GHz}-2.56 \mathrm{GHz}$. The simulated isolation between the two filters keeps better than $31 \mathrm{~dB}$.

Figure 7 and Figure 8 depict the magnetic intensity distributions at frequency channels of $1.855 \mathrm{GHz}$ and $2.505 \mathrm{GHz}$, respectively, based on Sonnet EM simulator.
The magnetic intensities with red colour regions in left and right sides for each figure stand for the effective microstrip resonator under each operating frequency channel, respectively. Accordingly, the maximum current intensities are 24 and 20 Ampere/Meter for the bandpass filter parts in left and right sides, respectively.

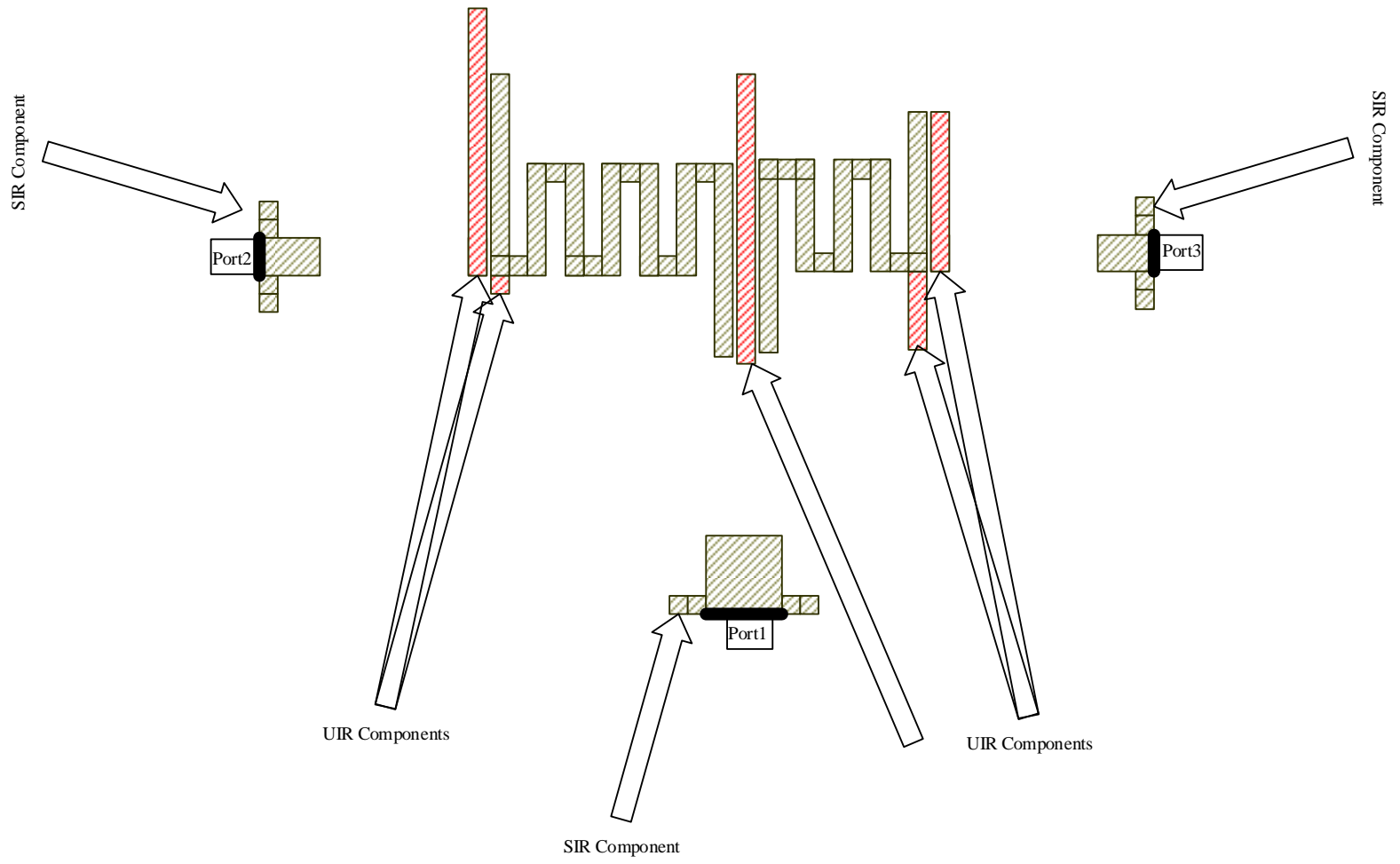

Fig. 4. Illustration of proposed diplexer elements before assembling.

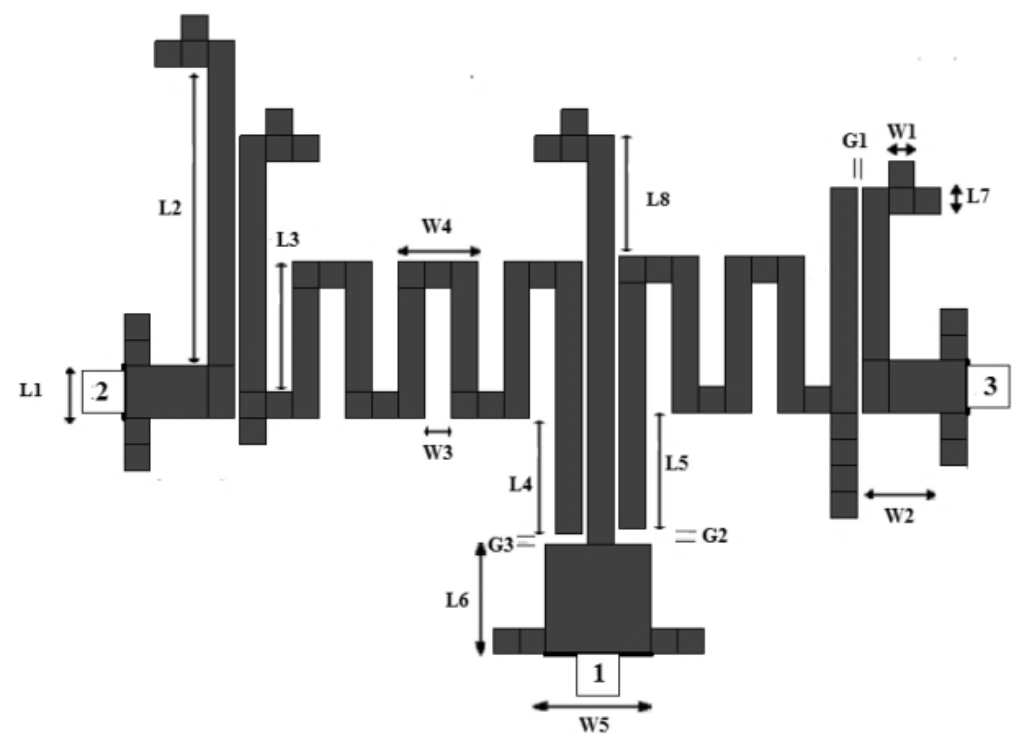

Fig. 5. The microstrip diplexer structure using meandered line resonator, SIRs, and UIRs after assembling. The diplexer dimensions (in mm units) are as follows: $\mathrm{W} 1=1, \mathrm{~W} 2=3, \mathrm{~W} 3=1, \mathrm{~W} 4=3, \mathrm{~W} 5=4, \mathrm{~L} 1=2, \mathrm{~L} 2=11.4, \mathrm{~L} 3=5, \mathrm{~L} 4=4.2, \mathrm{~L} 5=4.4, \mathrm{~L} 6=4.2, \mathrm{~L} 7=1, \mathrm{~L} 8=4.6, \mathrm{G} 1=0.2, \mathrm{G} 2=0.6, \mathrm{G} 3=0.4$. 


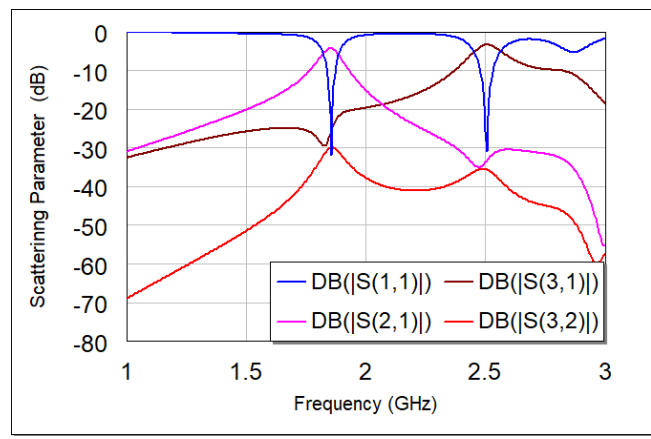

Fig. 6. Simulation results for projected diplexer based on FR4 substrate.
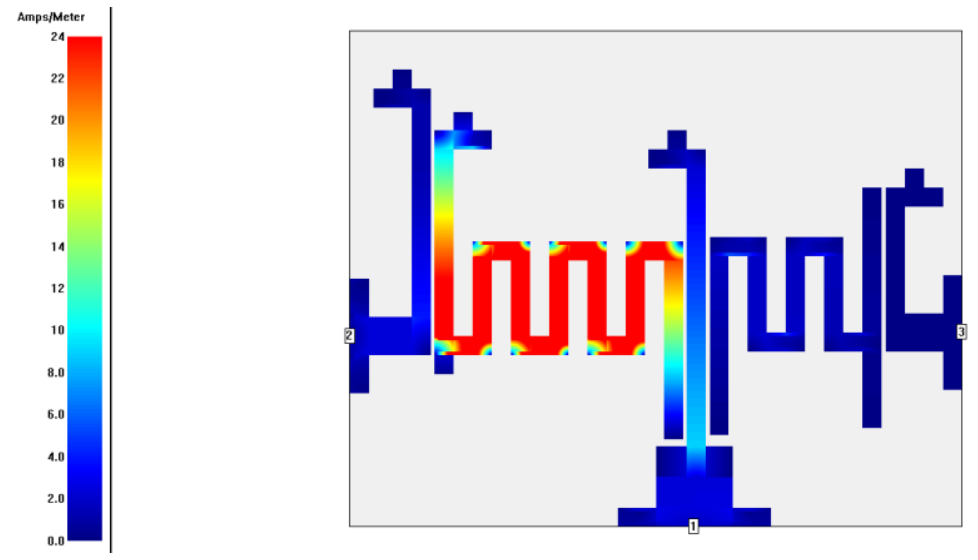

Fig. 7. The magnetic intensity distributions @ 1.855 GHz.
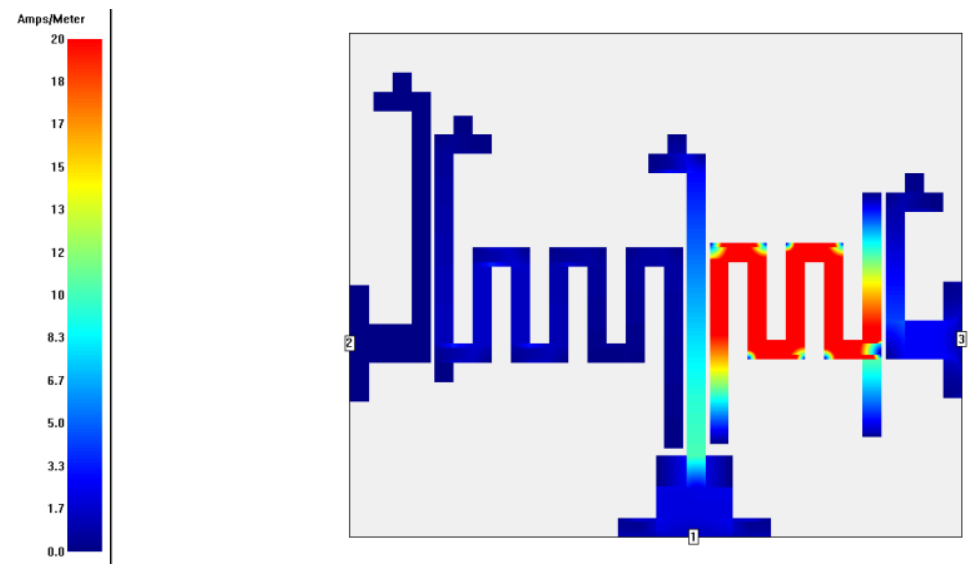

Fig. 8. The magnetic intensity distributions@ $2.505 \mathrm{GHz}$.

Figure 9 depicts the group delay (GD) performances for S11, S21, S31, and S32 scattering parameters. There are positive and negative pulses in GD response that specify the signal delay amounts in the diplexer. In fact, the negative GD does not violate causality; it only implies that the diplexer predicts where the pulse will be based on where it is now in the future. Recently, the RF and microwave devices, including filters [21], have been of great interest in the negative group delay (NGO) response. At the frequency channels of $1.855 \mathrm{GHz}$, and $2.505 \mathrm{GHz}$, the GD values are $46.1 \mathrm{~ns}$ and $-35.6 \mathrm{~ns}$, respectively. Moreover, the significant negative GD values are appeared at $1.83 \mathrm{GHz}$ with a value of $-3.62 \mathrm{~ns}$ for $\mathrm{S} 31$, at $2.74 \mathrm{GHz}$ and $3 \mathrm{GHz}$ with values of $1.7 \mathrm{~ns}$ and $-5.71 \mathrm{~ns}$ for $\mathrm{S} 21$, and at $2.965 \mathrm{GHz}$ with a value of -3.71 ns for $\mathrm{S} 32$.

The developed miniaturized microstrip diplexer using FR4 substrate in this paper has been compared with developed microstrip diplexers in [11], [16]-[20] as explained by Table I. The superiority of the proposed diplexer mostly comes from the high compactness, excellent return loss values, and the highest band isolation as compared with the reported diplexers in [11], [16]-[20]. The minor tolerable disadvantage of the designed device is that it has an insertion loss of $4.2 \mathrm{~dB}$ for channel 1 and $3.3 \mathrm{~dB}$ for channel 2, that are attributable to the dielectric loss and are comparable to the diplexer reported in [22].

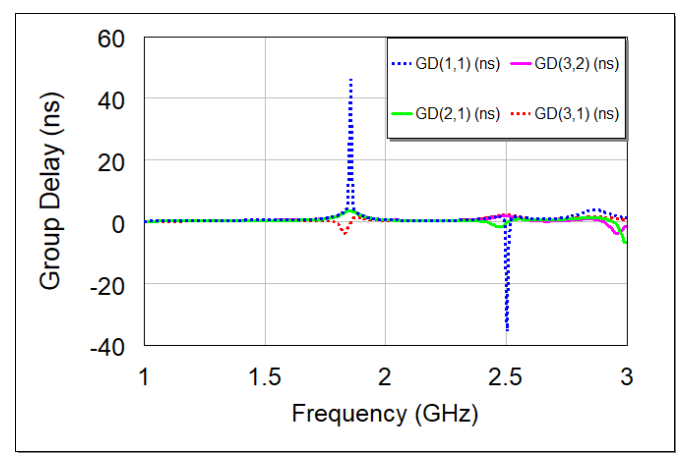

Fig. 9. The GD results for various scattering parameters. 
TABLE I. COMPARING THE DIPLEXER IN THIS STUDY WITH THE OTHERS REPORTED ONES IN THE LITERATURE.

\begin{tabular}{|c|c|c|c|c|c|}
\hline Reference & $\begin{array}{c}\text { Tx/Rx Resonances in } \\
(\mathbf{G H z})\end{array}$ & Insertion loss (dB) & Return loss (dB) & Isolation (dB) & Size (mm $\left.{ }^{2}\right)$ \\
\hline $\begin{array}{c}{[11]} \\
\text { without DGS }\end{array}$ & $1.58 / 2.38$ & $2 / 1.9$ & $17 / 21$ & 21.5 & $37 \times 21$ \\
\hline $\begin{array}{c}{[11]} \\
\text { with DGS }\end{array}$ & $1.2 / 1.6$ & $2.4 / 1.7$ & $19 / 25$ & 20 & $37 \times 21$ \\
\hline$[16]$ & $1.7 / 2.5$ & $2.35 / 1.96$ & $31 / 45.8$ & $>21$ & $32 \times 25$ \\
\hline$[17]$ & $1.75 / 2.35$ & $1.34 / 1.44$ & $\begin{array}{c}\text { Better than } 15 \mathrm{~dB} \text { for } \\
\text { both channels }\end{array}$ & 25 & $34 \times 26$ \\
\hline$[18]$ & $1.5 / 2$ & $2.7 / 2.8$ & $20 / 20$ & 40 & $33.9 \times 54.4$ \\
\hline$[19]$ & $2.4 / 3.2$ & $1.7 / 1.38$ & $30 / 30$ & 23 & $16 \times 47$ \\
\hline$[20]$ & $1.95 / 2.14$ & $1.22 / 1.12$ & $15 / 15$ & 30 & $40 \times 70$ \\
\hline Prop. & $1.855 / 2.505$ & $4.2 / 3.3$ & $32 / 31$ & 31 & $32 \times 26$ \\
\hline
\end{tabular}

\section{FABRICATION AND MEASUREMENT}

The snapshot of the fabricated microstrip diplexer is illustrated in Fig. 10.

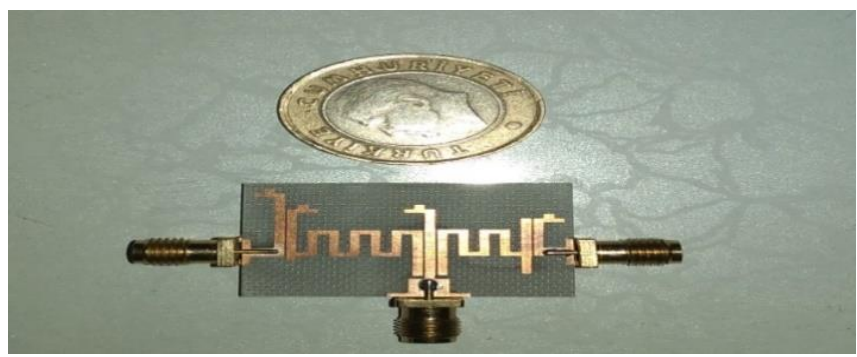

Fig. 10. Fabricated diplexer prototype using FR4 substrate.

The HP N9923A 6GHz Handheld Vector Network Analyzer (VNA) has been utilized to test the diplexer and validate the design simulations to corroborate it to be worked appropriately. The simulated and measured frequency responses for the projected diplexer are illustrated in Fig. 11 and Fig. 12.

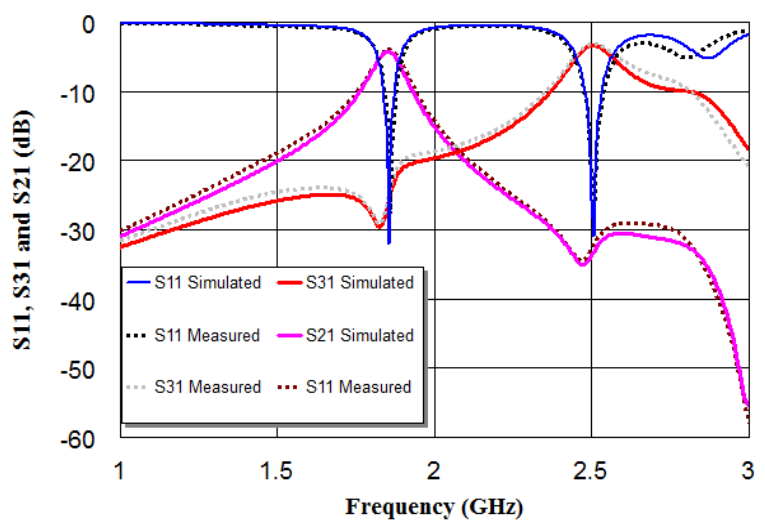

Fig. 11. Simulated and measured results for the proposed diplexer in terms of S11, S21, S31 scattering parameters.

The simulated and measured responses are consistent with each other. Noticeably, there are tolerable discrepancies between them attributable to slight discrepancy in diplexer dimensions, SMA soldering along with the influence of coaxial cable losses throughout the setup with VNA.

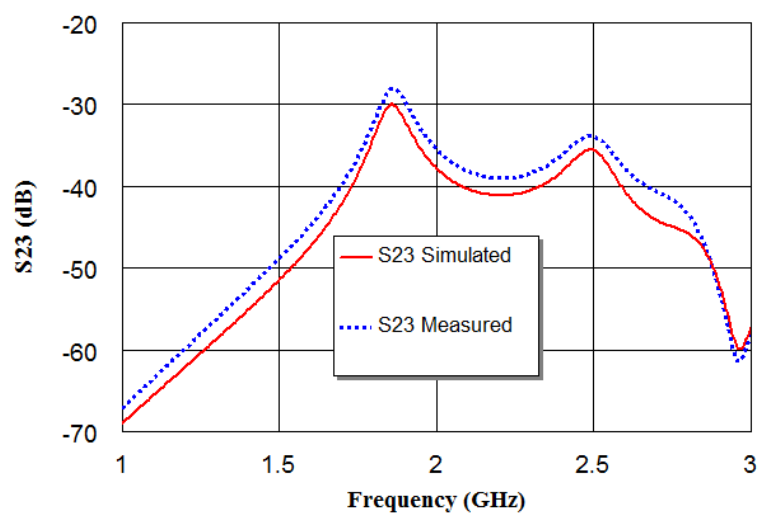

Fig. 12. Simulated and measured results for the proposed diplexer in terms of S32 scattering parameter (bands isolation).

\section{CONCLUSIONS}

In this study, a small microstrip diplexer is simulated with dual channels and very narrow bandwidths and successfully verified by the fabrication and measurements. The dual BPFs operating at the dual frequency bands of $1.855 \mathrm{GHz}$ and $2.505 \mathrm{GHz}$ and a coupled junction have been adopted to produce this diplexer. Every filter has been constructed by meandered line resonator, SIR elements, UIR elements, and three feed lines. The AWR solver has been employed to identify the response of the diplexer. The microstrip diplexer has been operated under $1.855 / 2.505 \mathrm{GHz}$ resonances for $\mathrm{Tx} / \mathrm{Rx}$ channels with isolation of bands better than $31 \mathrm{~dB}$. The proposed diplexer has very good electrical performances and a compact size of $32 \times 26 \mathrm{~mm}^{2}$ that are feasibly incorporated within many wireless configurations.

\section{ACKNOWLEDGMENT}

We acknowledge Dr. Ghassan N. Jawad from University of Baghdad for his support in providing the measured consequences.

\section{CONFLICTS OF INTEREST}

The authors declare that they have no conflicts of interest. 


\section{REFERENCES}

[1] T. Skaik, "Synthesis of coupled resonator circuits with multiple outputs using coupling matrix optimization", Ph.D. dissertation, University of Birmingham, 2011. DOI: 10.1049/iet-map.2010.0447.

[2] A. O. Nwajana and K. S. K. Yeo, "Microwave diplexer purely based on direct synchronous and asynchronous coupling", Radioengineering, vol. 25, no. 2, pp. 247-252, Jun. 2016. DOI: 10.13164/re.2016.0247.

[3] W. Kasparek, M. Petelin, V. Erckmann, A. Bruschi, F. Noke, F. Purps, F. Hollmann, Y. Koshurinov, L. Lubyako, B. Plaum, and W. Wubie, "High-power microwave diplexers for advanced ECRH systems", Fusion Engineering and Design, vol. 84, nos. 2-6, pp. 1002-1005, Jun. 2009. DOI: 10.1016/j.fusengdes.2008.11.070.

[4] H. Ezzeddine, S. Bila, S. Verdeyme, F. Seyfert, and D. Pacaud, "Coupling topologies for realizing compact microwave diplexers with dual-mode cavities", in Proc. of 2010 IEEE MTT-S International Microwave Symposium, 2010, pp. 880-883. DOI: 10.1109/MWSYM.2010.5517504.

[5] A. Yatongchai, R. Wongsan, and M. Krairiksh, "Design of a novel microwave diplexer using slit-top mushroom-like units electromagnetic band gap structure and transmission line layer", in Proc of The 8th Electrical Engineering/Electronics, Computer, Telecommunications and Information Technology (ECTI) Association of Thailand-Conference 2011, 2011, pp. 252-255. DOI: 10.1109/ECTICON.2011.5947820.

[6] M. Nath, "Design and characterization of microstrip diplexer", International Journal of Engineering Trends and Technology (IJETT), vol. 3, no. 2, pp. 183-186, 2012.

[7] T. Skaik and M. AbuHussain, "Design of diplexers for E-Band communication systems", in Proc. of 2013 13th Mediterranean Microwave Symposium (MMS), 2013, pp. 1-4. DOI: 10.1109/MMS.2013.6663087.

[8] W.-H. Tu, W.-C. Hung, and T.-H. Du, "Design of microwave microstrip multiband diplexers for system in package", IEEE Transactions on Components, Packaging and Manufacturing Technology, vol. 5, no. 4, pp. 502-507, Apr. 2015. DOI: 10.1109/TCPMT.2015.2409120.

[9] A. O. Nwajana and K. S. K. Yeo, "Multi-coupled resonator microwave diplexer with high isolation", in Proc. of 2016 46th European Microwave Conference (EuMC), 2016, pp. 1167-1170. DOI: 10.1109/EuMC.2016.7824556.

[10] H. S. Farahani, R. A. Sadeghzadeh, N. Gharanfeli, and A. Kishk, "Novel design of microwave diplexers using gap waveguide technology", Microwave and Optical Technology Letters, vol. 59, no. 5, pp. 1133-1136, May 2017. DOI: 10.1002/mop.30477.

[11] A. Chinig and H. Bennis, "A novel design of an H-shaped microstrip diplexer", Journal of Microwaves, Optoelectronics and
Electromagnetic Applications, vol. 16, no. 4, pp. 966-981, Dec. 2017 DOI: 10.1590/2179-10742017V16I4968.

[12] A. O. Nwajana, A. Dainkeh, and K. Yeo, "Substrate integrated waveguide (SIW) diplexer with novel input/output coupling and no separate junction", Progress in Electromagnetics Research, vol. 67, pp. 75-84, Mar. 2018. DOI: 10.2528/pierm18021603.

[13] G. M. Aristarkhov and I. N. Kirillov, "Microwave diplexers based on two- and three-resonators microstrip structures", in Proc. of 2019 Systems of Signals Generating and Processing in the Field of on Board Communications, 2019, pp. 1-4. DOI: 10.1109/SOSG.2019.8706806.

[14] Y. S. Mezaal, S. A. Hashim, A. H. Al-fatlawi, and H. A. Hussein, "New microstrip diplexer for recent wireless applications", International Journal of Engineering \& Technology, vol. 7, no. 3.4, article ID 16754, 2018. DOI: 10.14419/ijet.v7i3.4.16754.

[15] A. Singh and Rajni, "Stepped impedance resonator and spiral resonator based metamaterial unit-cell", Journal of Engineering Science \& Technology Review, vol. 9, no. 3, pp. 134-137, 2016. DOI: 10.25103/jestr.093.20.

[16] A. Chinig, "A novel design of microstrip diplexer using meander-line resonators", International Journal of Electronic Engineering and Computer Science, vol. 2, no. 2, pp. 5-10, 2017.

[17] T. Yang, P.-L. Chi, and T. Itoh, "Compact quarter-wave resonator and its application to miniaturized diplexer and triplexer", IEEE Transactions on Microwave Theory and Techniques, vol. 59, no. 2, pp. 260-269, 2011. DOI: 10.1109/TMTT.2010.2095029.

[18] C.-F. Chen, T.-Y. Huang, C.-P. Chou, and R.-B. Wu, "Microstrip diplexers design with common resonator sections for compact size, but high isolation", IEEE Trans. Microw. Theory Tech., vol. 54, no. 5 , pp. 1945-1952, 2006. DOI: 10.1109/TMTT.2006.873613.

[19] A. Chinig, J. Zbitou, A. Errkik, L. E. Abdellaoui, A. Tajmouati, M. Latrach, and A. Tribak,"A novel design of microstrip diplexer using triangular loop resonators", International Journal of Microwave and Optical Technology, vol. 10, no. 1, pp. 40-47, Jan. 2015.

[20] M.-L. Chuang and M.-T. Wu, "Microstrip diplexer design using common T-shaped resonator", IEEE Microwave and Wireless Components Letters, vol. 21, no. 11, pp. 583-585, 2011. DOI: 10.1109/LMWC.2011.2168949.

[21] G. Chaudhary, Y. Jeong, and J. Lim, "Microstrip line negative group delay filters for microwave circuits", IEEE Transactions on Microwave Theory and Techniques, vol. 62, no. 2, pp. 234-243, 2014. DOI: 10.1109/TMTT.2013.2295555.

[22] T. Yang and G. M. Rebeiz, "Three-pole 1.3-2.4-GHz diplexer and 1.1-2.45-GHz dual-band filter with common resonator topology and flexible tuning capabilities", IEEE Trans. Microw. Theory Techn., vol. 61, no. 10, pp. 3613-3624, 2013. DOI: 10.1109/TMTT.2013.2279381.

This article is an open access article distributed under the terms and conditions of the Creative Commons Attribution 4.0 (CC BY 4.0) license (http://creativecommons.org/licenses/by/4.0/). 